\title{
Direct Numerical Simulation of Turbulent Trailing-Edge Flow with Base Flow Control
}

\author{
Y. F. Yao* and N. D. Sandham ${ }^{\dagger}$ \\ Southampton University, Southampton, England SO17 1BJ, United Kingdom
}

\begin{abstract}
Direct numerical simulation has been carried out for turbulent flow over a rectangular trailing edge at a Reynolds number of $1 \times 10^{3}$ (based on the freestream quantities and the trailing-edge thickness) and ratio of boundarylayer displacement thickness to trailing-edge thickness close to unity. Two types of flow control were studied: base transpiration and secondary splitter plate. Simulation of base transpiration was performed using different slit heights and volume flow rates. It was found that even small flow rates could produce significant changes in overall aerodynamic performance, measured, for example, by the base pressure coefficient. It was also found that for the same volume flow rate, a greater increase in base pressure (drag reduction) was obtained by blowing slowly through a wide slit rather than quickly through a narrow slit. The effectiveness of a secondary splitter plate located on the trailing-edge centerline was investigated by varying the plate length from one to five times the trailing-edge thickness. A significant increase in the base pressure coefficient (about $25 \%$ ) was achieved, even with the shortest splitter plate equal to the trailing-edge thickness. The base pressure coefficient increased monotonically with the splitter plate length, and no intermediate maximum value was found.
\end{abstract}

$\left(C_{p}\right)_{b}$
$h_{s}$
$k$
$L_{\mathrm{sp}}$
$n$
$p$
$p_{b}$
$p_{\text {ref }}$
$q_{b}$
$R_{h}$
$R_{\delta^{*}}$
$t$
$U$
$u_{b}$
$u_{i}(u, v, w)$
$u_{r}$
$u_{\tau}$
$x_{i}(x, y, z)$
$x_{r}$
$y^{+}$
$\Delta t$
$\Delta x_{i}^{+}$
$\delta_{99}$
$\delta^{*}$
$\delta_{\text {in }}^{*}$

$\quad$ Nomenclature
$=$ base pressure coefficient
$=$ base slit height (width)
$=$ turbulence kinetic energy
$=$ length of secondary splitter plate
$=$ current time step
$=$ instantaneouspressure
$=$ base pressure
$=$ reference pressure
$=$ base volume flow rate
$=$ Reynolds number based on freestream
$=$ velocity and trailing-edge thickness
$=$ Reynolds number based on freestream
$=$ velocity and displacement thickness
$=$ time variable
$=$ streamwise mean velocity
$=$ base transpiration velocity
$=$ instantaneous velocity variables
$=$ friction velocity, $\left[(\mathrm{d} u / \mathrm{d} y)_{w} / R_{h}\right]^{1 / 2}$
$=$ Cartesian coordinate axes
$=$ recirculation length at centerline
$=y$ coordinate in wall units, $y u_{\tau} R e_{h}$
$=$ increment of time step
$=$ increments of coordinates $x_{i}$ in wall units
$=$ boundary-layerthickness
$=$ boundary-layerdisplacement thickness
$=$ boundary-layerdisplacement thickness at inlet

\section{Introduction}

$\mathbf{T}$ HE trailing-edgeregion of airfoils and turbine blades influences the entire aerodynamic performance, measured, for example,

\footnotetext{
Received 23 August 2001; revision received 28 March 2002; accepted for publication 28 March 2002. Copyright (C) 2002 by Y. F. Yao and N. D. Sandham. Published by the American Institute of Aeronautics and Astronautics, Inc., with permission. Copies of this paper may be made for personal or internal use, on condition that the copier pay the $\$ 10.00$ per-copy fee to the Copyright Clearance Center, Inc., 222 Rosewood Drive, Danvers, MA 01923; include the code 0001-1452/02 \$10.00 in correspondence with the CCC.

* Research Fellow, Aeronautics and Astronautics, School of Engineering Sciences; y.yao@soton.ac.uk.

${ }^{\dagger}$ Professor, Aeronautics and Astronautics, School of Engineering Sciences; n.sandham@soton.ac.uk.Senior Member AIAA.
}

by the base pressure coefficient or total pressure loss. Because the region is highly localized, it can be a good candidate for the introduction of flow control techniques aiming at drag reduction. $\mathrm{Nu}$ merically, it is difficult to achieve an accurate simulation using the conventional Reynolds-averaged Navier-Stokes approach because of the complexity of the problem. In the immediate vicinity of the trailing edge, the flow may be highly unsteady and far from an equilibrium state, and, consequently, conventional turbulence models either prove to be inadequate or require special modifications. However, simplified versions of the problem can be computed using direct numerical simulation (DNS). Previous experience ${ }^{1}$ showed that good quality results can be obtained at moderate Reynolds number $\left(\sim 1 \times 10^{3}\right.$ based on the trailing-edgethickness or boundarylayer displacement thickness) with currently available computer resources. The intention of this paper is, therefore, to study mechanisms of flow control in a model trailing-edge flow using DNS.

Early research work on the application of flow control to the trailing edge was carried out by Nash, ${ }^{2}$ Wood, ${ }^{3}$ and Bearman ${ }^{4}$ around the mid-1960s, when studies were made of the effects of base bleed (also called secondary airflow) on the aerodynamic performance of the two-dimensional blunt trailing edge, in particular, the increase in base pressure coefficient (and consequent decrease in drag coefficient). Since then, the base bleed concept has been applied to a wider range of practical problems, for example in transonic and supersonic flows. ${ }^{5}$ In the gas-turbine industry, a similar technique called gas coolant ejection has also been developed and used for the control of turbine blade temperature to extend the lifetime of blades. Experiments have shown that the method not only results in a lower heat transfer to the blade, but also leads to a significant reduction in total pressure loss. ${ }^{6}$ When the ejection velocity and angle were tuned, an overall optimization could be achieved. Although the method clearly works, it is still important to understand the underlying flow mechanism. On this point, experimentalists have made some progress, and the mechanism of drag reduction is believed to involve a change in the wake dynamics. ${ }^{8}$ Computational studies have progressed with improvements in computer power and numerical methods. Hannemann and Oertel ${ }^{9}$ tackled the laminar wake unsteadiness problem, and Hammond and Redekopp ${ }^{10}$ analyzed the global instability of the flow. These pilot investigations showed that the global dynamics associated with vortex shedding could be suppressed by base transpiration. Based on the success of these laminar calculations, a fully turbulent flow study is desirable.

An alternative way of achieving the base drag reduction is to use an additional (secondary) splitter plate along the wake centerline. An important theoretical investigation of the effect of such a splitter plate on the drag and pressure distribution of bluff body flow 
was carried out by Roshko. ${ }^{11}$ Later, this simple but effective concept was applied in experiments, ${ }^{12,13}$ and a review paper was given by Tanner. ${ }^{14}$ With developments in experimental and computational techniques, there have been additional investigations in recent years. ${ }^{15,16}$ Compared to the blunt trailing-edge geometry, the presence of a splitter plate modifies the near-wake vortex evolution and, thus, limits the main vortex interactions in the recirculation zone. It has been confirmed both by experiments $\mathrm{s}^{12,13,15}$ and by two-dimensionalcomputation ${ }^{16}$ that the drag coefficient and vortex shedding can be significantly reduced by the use of a splitter plate of relatively short length.

The present study is focused on the two types of flow control just described. The computation includes a precursor simulation of turbulent boundary-layerflow and a successor simulation of turbulent trailing-edgeflow. Flow characteristics with no flow control are studied first, providing a benchmark for reference and illustrating the main features of the vortex shedding and interaction mechanism. Simulations with flow control using base transpiration and a secondary splitter plate follow, with emphasis on the base pressure coefficient, flow structure, and mean properties.

\section{Simulation}

\section{Governing Equations and Numerical Method}

All variables defined in the Nomenclature are nondimensional. Distances are normalized with trailing-edge thickness, velocities with freestream velocity, and pressure with freestream density times the square of freestream velocity.

We consider an incompressible fluid moving with velocity $u_{i}=(u, v, w)$ and pressure $p$ in a Cartesian coordinate system $x_{i}=(x, y, z)$ and use $t$ to denote the time. The fluid motion satisfies the dimensionless Navier-Stokes equations given by

$$
\frac{\partial u_{i}}{\partial t}+\frac{\partial u_{j} u_{i}}{\partial x_{j}}=-\frac{\partial p}{\partial x_{i}}+\frac{1}{R e_{h}} \frac{\partial^{2} u_{i}}{\partial x_{j} \partial x_{j}}
$$

and the continuity equation

$$
\frac{\partial u_{i}}{\partial x_{i}}=0
$$

The Reynolds number $R e_{h}$ is based on the dimensional reference velocity (chosen as the freestream velocity) and the dimensional reference length (chosen as the trailing-edgethickness), which have been used throughout this paper to make all variablesdimensionless.

The Navier-Stokes equations are discretized on a staggered grid using a second-ordercentral finite difference scheme and advanced in time with the projection method based on a second-orderexplicit Adams-Bashforth scheme. The provisionalvelocity $u_{i}^{*}$ is projected using the data from the current time step $n$ and preceding time step $n-1$ as

$$
\frac{u_{i}^{*}-u_{i}^{n}}{\Delta t}=\frac{3}{2} H_{i}^{n}-\frac{1}{2} H_{i}^{n-1}+\frac{1}{2} \frac{\partial p^{n-1}}{\partial x_{i}}
$$

where the quantity $H_{i}$ is defined as

$$
H_{i}=-\frac{\partial u_{j} u_{i}}{\partial x_{j}}+\frac{1}{R e_{h}} \frac{\partial^{2} u_{i}}{\partial x_{j} \partial x_{j}}
$$

This is then corrected for continuity to yield the velocity at a time step $n+1$ using

$$
u_{i}^{n+1}=u_{i}^{*}-\frac{3}{2} \Delta t \frac{\partial p^{n}}{\partial x_{i}}
$$

where the pressure $p^{n}$ is obtained by the solution of the Poisson equation

$$
\frac{\partial^{2} p^{n}}{\partial x_{i} \partial x_{i}}=\frac{2}{3 \Delta t} \frac{\partial u_{i}^{*}}{\partial x_{i}}
$$

\section{Turbulent Boundary-Layer Flow}

Simulation of turbulent boundary-layerflow was performed first, using a spatial DNS code that implemented the rescaling and feedback technique developed by Lund et al. ${ }^{17} \mathrm{~A}$ computation box of size $50 \times 20 \times 6$ (based on units of inlet displacement thickness $\left.\delta_{\text {in }}^{*}\right)$ in the streamwise $x$, wall-normal $y$, and spanwise $z$ directions was used, with $128 \times 96 \times 64$ grid points, stretched in the wallnormal direction and uniformly distributed in the streamwise and spanwise directions. A grid resolution of $\Delta x^{+}=19, \Delta y_{1}^{+}=0.5$, and $\Delta z^{+}=4.5$ was achieved with about 10 points in the viscous sublayer $\left(y^{+} \leq 10\right)$. The turbulent boundary layer was simulated with a condition of $R e_{\delta^{*}}=950$ at the inflow plane. Results for skin-friction and turbulence statistics were in good agreement with other DNS data and experimental results. ${ }^{1}$ A time sequence of flow variables at a sampling plane near the exit was stored for later use as the inflow boundary condition in the following turbulent trailing-edge simulation.

\section{Turbulent Trailing-Edge Flow}

Simulations of turbulent flow over a rectangulartrailing-edge geometry were carried out at a Reynolds number $R e_{h}=1 \times 10^{3}$. The upper and lower incoming turbulent boundary-layers were statistically equivalent but uncorrelated in time. They developed along the plate for a short distance before contacting each other at the trailing edge. The Cartesian coordinate system was built up with its origin $O$ at the center of the trailing edge.

Computations were carried out with a parallel complex-geometry simulation code (see Thomas and Williams ${ }^{18}$ ) that has been parallelized using the message passing interface (MPI) library and ported to the Cray-T3E parallel computer. Validations have been made for various cases such as backward-facing step flow, openchannel flow, and skewed flow past a wall-mounted cube, all giving good agreement with published computational results and experimental data.

The computational box size is $20 \times 16 \times 6$, with the $x$ length of 20 split into 5 along the flat plate and 15 in the wake region in the streamwise $x$ direction. The upstream distance of 5 is chosen based on the experiment observation by Gough and Hancock ${ }^{19}$ that the boundary layer more than $\delta_{99}$ ahead of the trailing edge is unaffected by the presence of the trailing edge, as well as the numerical study by Hannemann and Oertel $^{9}$ that the rms fluctuations at this location were more than two orders of magnitude lower than the maximum rms fluctuations occurring in the wake. Lengths of 8.5 on the upper side and 7.5 on the lower side in the wall-normal $y$ directionare used, whereas the length in the spanwise $z$ direction is 6 (Fig. 1). This asymmetric arrangement in $y$ was chosen mainly on the grounds of load balancing on the parallel computer used in this study but also as a check on the box size because any asymmetries in the results would imply that the computational box was too small in the $y$ direction. Grid points are uniformly distributed in all three directions with $256 \times 512 \times 64$ points in $x, y$, and $z$, respectively.A detailed study of domain size dependency and grid resolution was carried out by Yao et al., ${ }^{1}$ with the conclusion that this configuration and grid resolution are suitable for the current simulations.

The boundary conditions are defined as follows. At the inlet, the data are prescribed and taken from the precursor turbulent

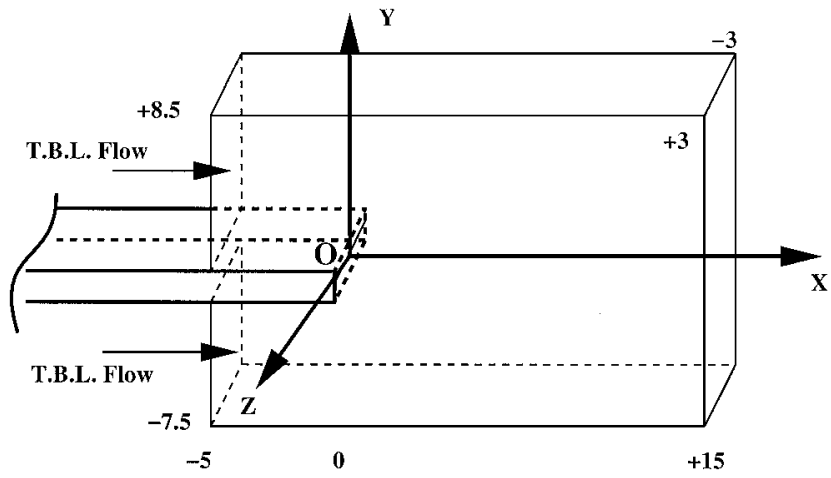

Fig. 1 Computational box for the trailing-edge simulation with and without flow control (not to scale) 


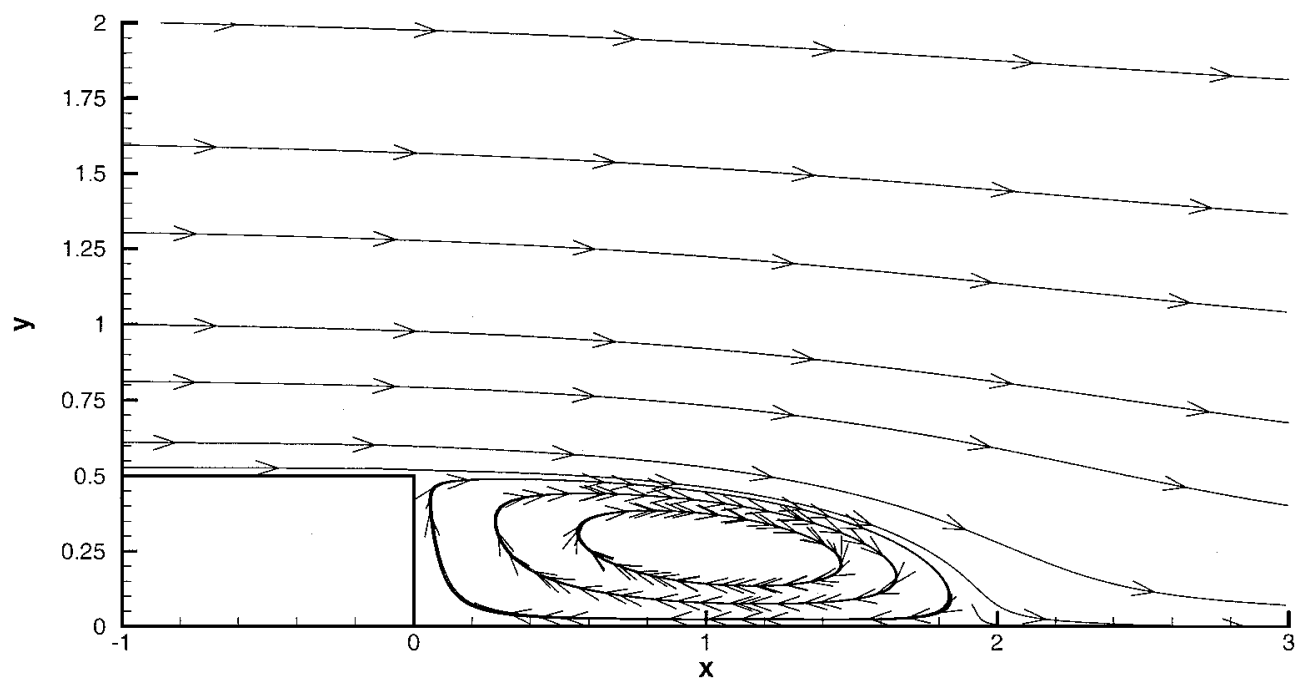

Fig. 2 Streamlines in the near-wake region with no flow control.

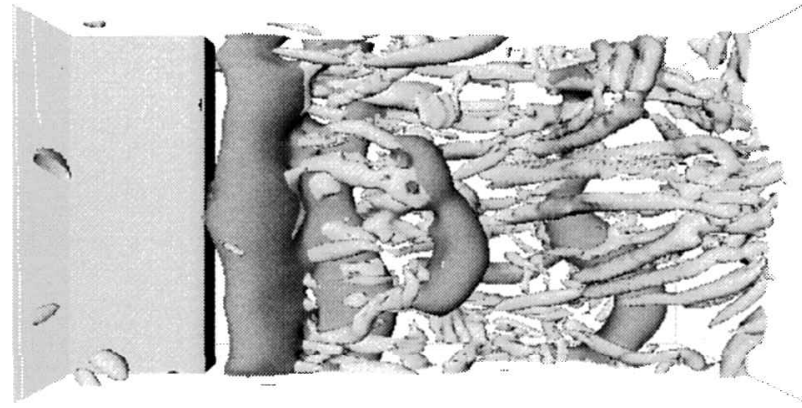

Top view

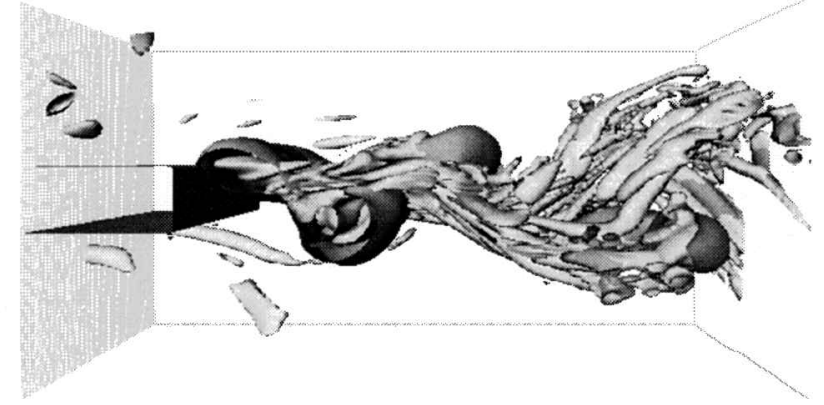

Side view

Fig. 3 Instantaneous flow structures in the near-wake region from the simulation with no flow control; spanwise vortices appear in the darker shade, showing a pressure isosurface with $p=-\mathbf{0 . 0 3 5}$, whereas streamwise vortices, in the lighter shade, show $\Pi$ vortices with $\Pi=0.4$.

boundary-layer simulation. The outlet plane is treated with a standard convective condition, so that outgoing disturbances can leave the domain smoothly. On the top and bottom boundaries of the domain, free-slip conditions are used, and in the spanwise direction a periodic condition is applied. A no-slip condition is used on all solid walls, including the splitter plate when present.

\section{Results and Discussion}

\section{Reference Case: No Flow Control}

For reference, we first give a brief overview of results for the case with no flow control. More complete sets of results for this case were reported by Yao et al. ${ }^{1}$ and Thomas et al. ${ }^{20}$ Figure 2 shows the streamline plots in the near wake. (Only one half-domain is plotted due to the symmetry.) A recirculationregion with a length of $x_{r} \simeq 2.0$ (where $x_{r}$ is defined as the dimensionless distance between the trailing-edge end plane and the stagnation point in the wake) exists after the trailing edge. The maximum reverse velocity $u_{r}$ is about $10 \%$ of the freestream velocity. The turbulence kinetic energy reaches its peak value of about 0.03 at the centerline in the near wake and then decays downstream. The base pressure coefficient is defined by

$$
\left(C_{p}\right)_{b}=2\left(p_{b}-p_{\text {ref }}\right)
$$

where the averaged dimensionless base pressure $p_{b}$ is calculated by integrating the static pressure over the trailing-edge end plane, and the reference dimensionless pressure $p_{\text {ref }}$ is taken as the freestream value at the inlet plane. The simulation (which itself uses a pressure relative to a fixed point on the solid wall upstream of the trailing edge) gives $p_{b}=-0.0695$ and $p_{\text {ref }}=-0.036$. The base pressure coefficient is, therefore, $-\left(C_{p}\right)_{b}=0.0669$.

Snapshots of an instantaneous flowfield are shown in Fig. 3 in a subdomain of $10 \times 6 \times 6$. This illustrates the interaction be-
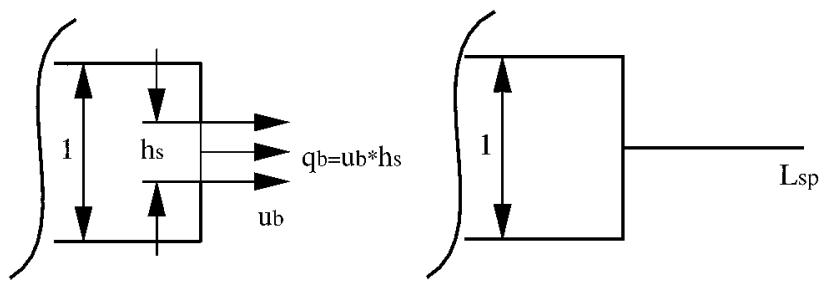

a)

b)

Fig. 4 Configurations of the two types of flow control: a) base transpiration and b) secondary splitter plate; the trailing-edge thickness is set to be 1 .

tween the large-scale spanwise vortices (presented in the darker shade, an isosurface of pressure containing a low-pressure core) and smaller-scale streamwise vortices (presented in the lighter shade, an isosurface of the second invariant of the velocity gradient tensor $\left[\Pi=\left(\partial u_{i} / \partial x_{j}\right)\left(\partial u_{j} / \partial x_{i}\right)\right]$, named $\Pi$ vortices for later use). It can be seen that the well-known von Kármán vortex street exists in the near wake. The appearance of vortex shedding is related (via a global mode ${ }^{10}$ ) to the existence of absolute instability based on linear stability theory (see Hannemann and Oertel ${ }^{19}$ ).

\section{Flow Control by Base Transpiration}

Simulations with base transpiration were carried out using the arrangement shown in Fig 4a. A total of 10 simulations (cases 110) were carried out with different slit heights $\left(h_{s}=0.25,0.5\right.$, and $1.0)$ and volume flow rates per unit span $\left(q_{b}= \pm 0.025, \pm 0.05\right.$, and \pm 0.1 ), defined as $q_{b}=u_{b} h_{s}$, where $u_{b}$ is the uniform base flow velocity. The particular combinations chosen for $h_{s}, u_{b}$, and the corresponding $q_{b}$ are shown in Table 1 .

The overall effectiveness of the flow control can be measured by the effect on the base pressure coefficient and the base pressure. 
These are plotted in Fig. 5. The near collapse of the curves, when plotted against volume flow rate in this manner, illustrates that volume flow rate is the key parameter. Base pressure drops sharply when suction is applied. The corresponding base pressure coefficient decreases (and consequently drag increases) by up to a factor of four for suction with volume flow rate $q_{b}=-0.1$. For base blowing, the opposite effect is observed, with a maximum increase of base pressure coefficient (drag reduction) of approximately a factor of two for $q_{b}=0.1$. However, for blowing, the effectiveness is strongly affected by slit width $h_{s}$, and it is clear that, for the same volume flow rate, a greater increase in base pressure coefficient is obtained by blowing slowly through a wide slit rather than quickly through a narrow slit. This is in good agreement with the experimental observation that the best drag reduction for a given mass flow can be obtained by ejecting the bleed air at the lowest possible velocity. ${ }^{3,14}$

The effect of base transpiration on the mean flow is revealed by streamline plots. Figure 6 shows the effect of varying the base transpiration through a slit height of $h_{s}=0.25$. Three cases are shown with $u_{b}=-0.1,0.1$, and 0.4 and, consequently, $q_{b}$ equal to -0.025 , 0.025 , and 0.1 . For weak suction flow, $q_{b}=-0.025$, we see that the recirculation length $x_{r}$ has reduced to about 1.58 , compared to 2.0 for the reference case of no flow control. The closed recirculation zone is located between the edge of the slit, $y=0.0125$, and the edge of the plate, $y=0.5$. For weak blowing flow, $q_{b}=0.025$, the main recirculation has moved downstream, starting at $x=1.0$ and ending at $x=2.48$, giving a total recirculation length of $x_{r}=1.48$. A secondary recirculation is also observed just above the slit, adjacent

Table 1 Simulations with two types of flow control: base transpiration and a secondary splitter plate

\begin{tabular}{lllllll}
\hline \hline Case & $h_{s}$ & $u_{b}$ & $q_{b}$ & $x_{r}$ & $u_{r}$ & $L_{\mathrm{sp}}$ \\
\hline Ref. & 0.0 & 0.0 & 0.0 & 2.0 & -0.1 & 0 \\
1 & 0.25 & -0.4 & -0.1 & 1.05 & -0.4 & - \\
2 & 0.25 & -0.1 & -0.025 & 1.58 & -0.124 & - \\
3 & 0.25 & +0.1 & +0.025 & 1.48 & -0.046 & - \\
4 & 0.25 & +0.4 & +0.1 & - & - & - \\
5 & 0.5 & -0.2 & -0.1 & 0.98 & -0.25 & - \\
6 & 0.5 & -0.1 & -0.05 & 1.45 & -0.154 & - \\
7 & 0.5 & +0.1 & +0.05 & - & - & - \\
8 & 0.5 & +0.2 & +0.1 & - & -0.2 & - \\
9 & 1.0 & -0.1 & -0.1 & 1.0 & -0.0849 & 1 \\
10 & 1.0 & +0.1 & +0.1 & - & -0.0805 & 2 \\
11 & - & - & 0.0 & 3.00 & -0.045 & 3 \\
12 & - & - & 0.0 & 3.34 & -0.0 & 4 \\
13 & - & - & 0.0 & 3.63 & -0.045 \\
14 & - & - & 0.0 & 3.91 & - & 5 \\
15 & - & - & 0.0 & 4.21 & - & \\
\hline \hline
\end{tabular}

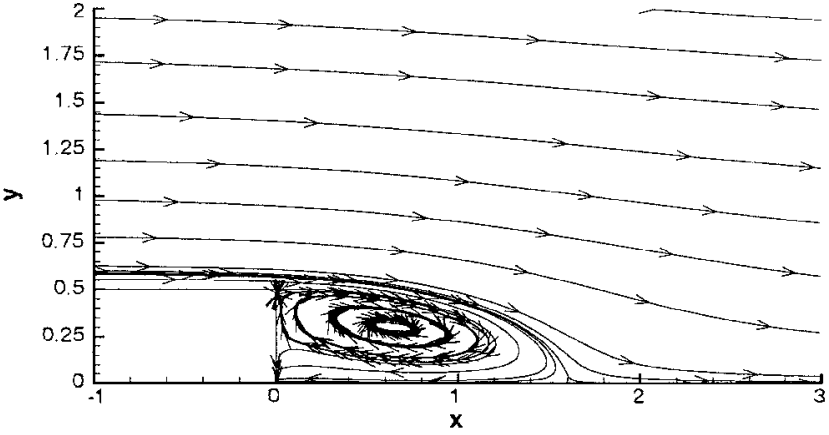

a)

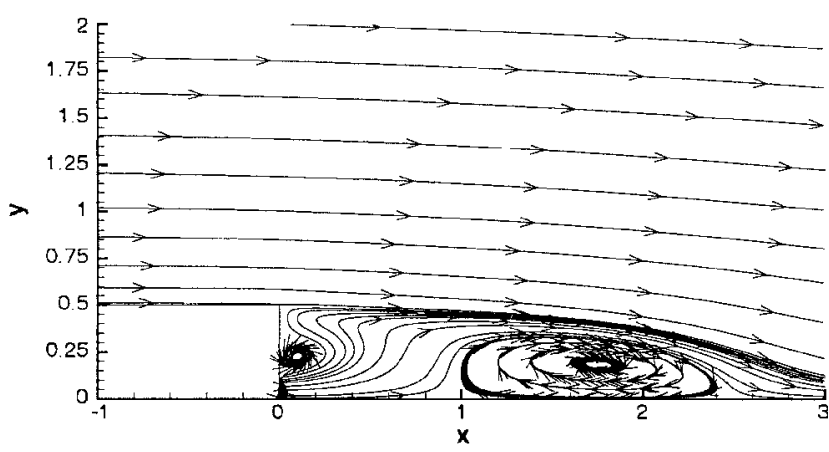

b)

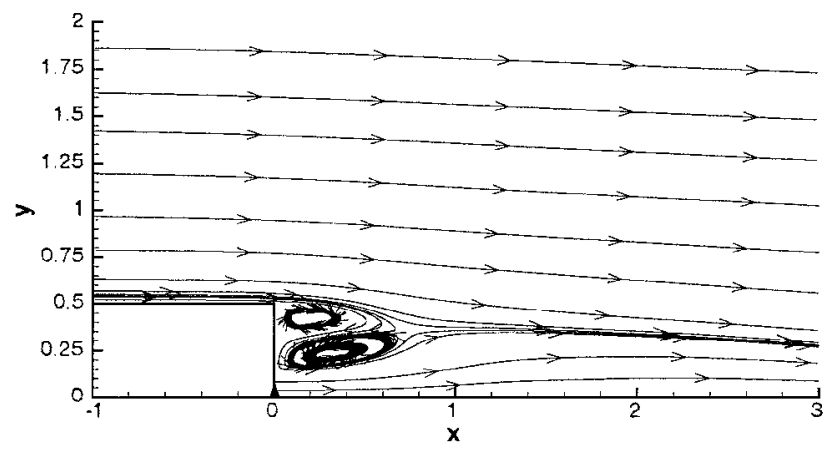

c)

Fig. 6 Streamlines for base transpiration through a slit of height $h_{s}=0.25$, with volume flow rates of $q_{b}:$ a) $\left.-0.025, \mathrm{~b}\right) 0.025$, and c) 0.1 .

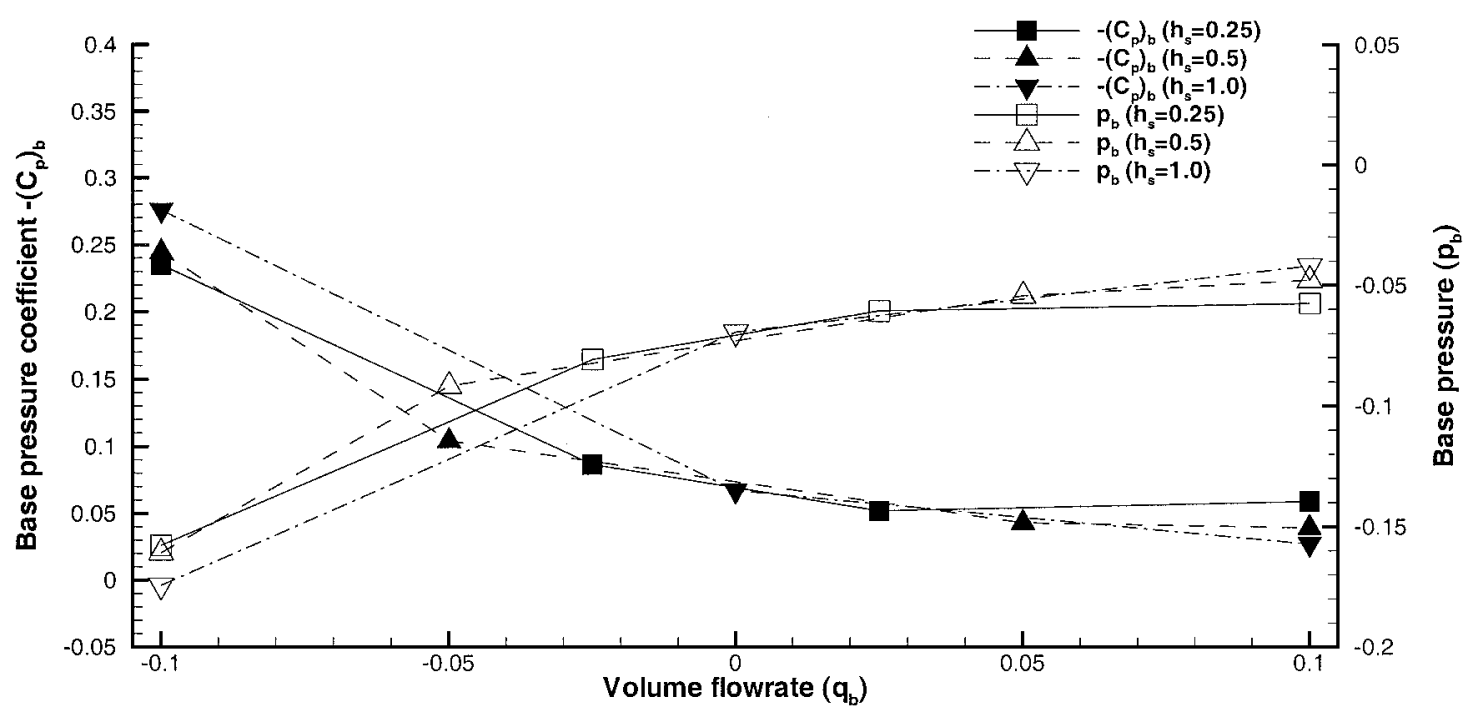

Fig. 5 Base pressure coefficient (left side, solid symbols) and base pressure (right-hand side, open symbols) as a function of volume flow rate. 
to the trailing edge. For very strong blowing flow, $q_{b}=0.1$, there is no downstream recirculation zone, but now two small recirculation zones are seen in the very near wake region, between the slit and the edge of the plate. Streamwise mean velocity $U$ and turbulence kinetic energy $k$ variations along the centerline $(y=0)$ are shown in Figs. 7 and 8 for all of the cases with $h_{s}=0.25$ and $h_{s}=0.5$, respectively. It can be seen that, in general, as $q_{b}$ is decreased, the recirculation zone (negative $U$ ) moves closer to the trailing edge, and the magnitude of the (negative) peak increases, accompaniedby an increase in $k$. From Figs. 7 and 8, it appears that, except for the case with the strongest suction, the peak value of $k$ occurs approximately twice as far from the trailing edge as the local minimum in $U$. As a summary, values of the maximum recirculation velocity $u_{r}$ and the recirculation length $x_{r}$ for all cases run are shown in Table 1 .

Instantaneous flow structures show the effect of base transpiration on the structure of the near wake. Figure 9 shows snapshots from the cases of strong suction $\left(h_{s}=1.0, u_{b}=-0.1\right.$, and $\left.q_{b}=-0.1\right)$ and strong blowing $\left(h_{s}=1.0, u_{b}=0.1\right.$, and $\left.q_{b}=0.1\right)$, illustrating the flow structure with isosurfaces of pressure and $\Pi$, as shown earlier for the reference case in Fig. 3. For the strong suction case, it can be seen that the quasi-two-dimensional coherent structure has been strengthened considerably. The strong spanwise vortex structures even appear upstream of the trailing edge. Subsequently, the $\Pi$ vortices also become stronger because of the interactions. Conversely, with strong blowing, the structures are pushed farther downstream and become much weaker. As a result, a smaller peak value of $k$ is evident (Figs. 7 and 8). There also seems to be tendency to lose the strong spanwise coherence of the pressure structures for the case with strong blowing, and the shedding has been moved farther downstream, agreeing qualitatively with the experiment. ${ }^{4}$

Suction or blowing also affects the skin friction on the plate upstream of the trailing edge, with suction increasing the friction drag and blowing decreasing it. However, this effect was at most only $9.7 \%$ of the base pressure drag change.

\section{Flow Control by Secondary Splitter Plate}

An alternative technique of flow control is the addition of a zerothickness secondary splitter plate behind the trailing edge as illustrated in Fig. 4b. In this study, the splitter plate length $L_{\mathrm{sp}}$ is varied over a range of $1-5$, and results are compared with those from the reference case of no flow control and experimental data.

Figure 10 gives the base pressure coefficient and base pressure variations as a function of splitter plate length $L_{\mathrm{sp}}$. With $L_{\mathrm{sp}}=5$, a maximum reduction of $-\left(C_{p}\right)_{b}$ of about $44 \%$ is achieved. However, a splitter plate with $L_{\mathrm{sp}}=1$ already gives a $25 \%$ reduction. Shorter plates are probably also preferred given practical (structural) considerations. A maximum drag reduction of $50 \%$ was achieved experimentally by Bearman ${ }^{13}$ for a flow with Mach number approximately 0.1 and splitter plate length $L_{\mathrm{sp}}=4$. However, compared to the results of Nash et al., ${ }^{12}$ who studied the secondary splitter plate for a rectangular trailing-edge geometry at Mach number 0.4 , there are large differences. The magnitudes of the reductions of $-\left(C_{p}\right)_{b}$ are here much smaller than those in the Nash et al. experiment, which found a factor of two reduction for $L_{\mathrm{sp}}=1$ and a factor of three reduction for $L_{\mathrm{sp}}=4$. One possible cause for the difference is the reference pressure, here taken as the freestream at the computational inflow upstream of the trailing edge, whereas in the experiments it is presumably taken farther away from the object. Other differencesare the Mach number (incompressible flow was assumed in the current computation) and Reynolds number (lower in the computations).

A phenomenon of a local maximum in the base pressure coefficient as a function of $L_{\mathrm{sp}}$ was observed experimentallyby Bearman ${ }^{13}$ at Reynolds numbers between $1.4 \times 10^{5}$ and $2.56 \times 10^{5}$ in a lowspeed wind tunnel. A peak appeared at $L_{\mathrm{sp}}=1.5$ and increased significantly with the decrease of Reynolds number, indicating a strong Reynolds number effect. Such a peak was also observed in the experiments of Nash et al. ${ }^{12}$ However, the phenomenon was not found in a recent experimentby Rathakrishnan ${ }^{15}$ using a rectangular cylinder in a low-speed wind tunnel (Reynolds numbers $0.58 \times 10^{5}$ and $\left.0.98 \times 10^{5}\right)$. The experiments showed a significant decrease in $-\left(C_{p}\right)_{b}$ for $L_{\mathrm{sp}}=1$ (about a $40 \%$ reduction), but the effect of $L_{\mathrm{sp}}$
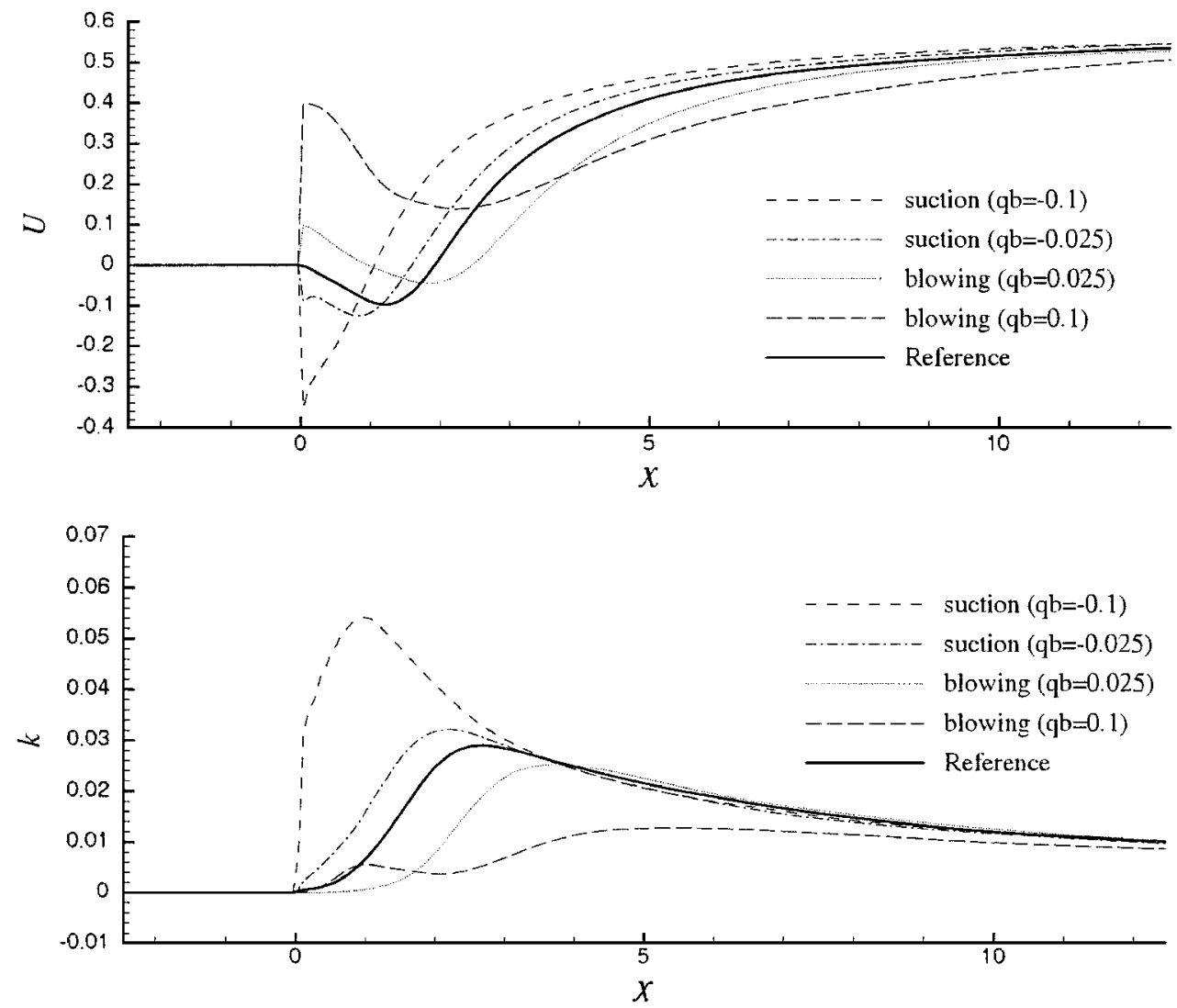

Fig. 7 Mean velocity $U$ and turbulence kinetic energy $k$ distributions for slit height $\boldsymbol{h}_{s}=0.25$; the reference simulation of no flow control is shown by the solid line. 

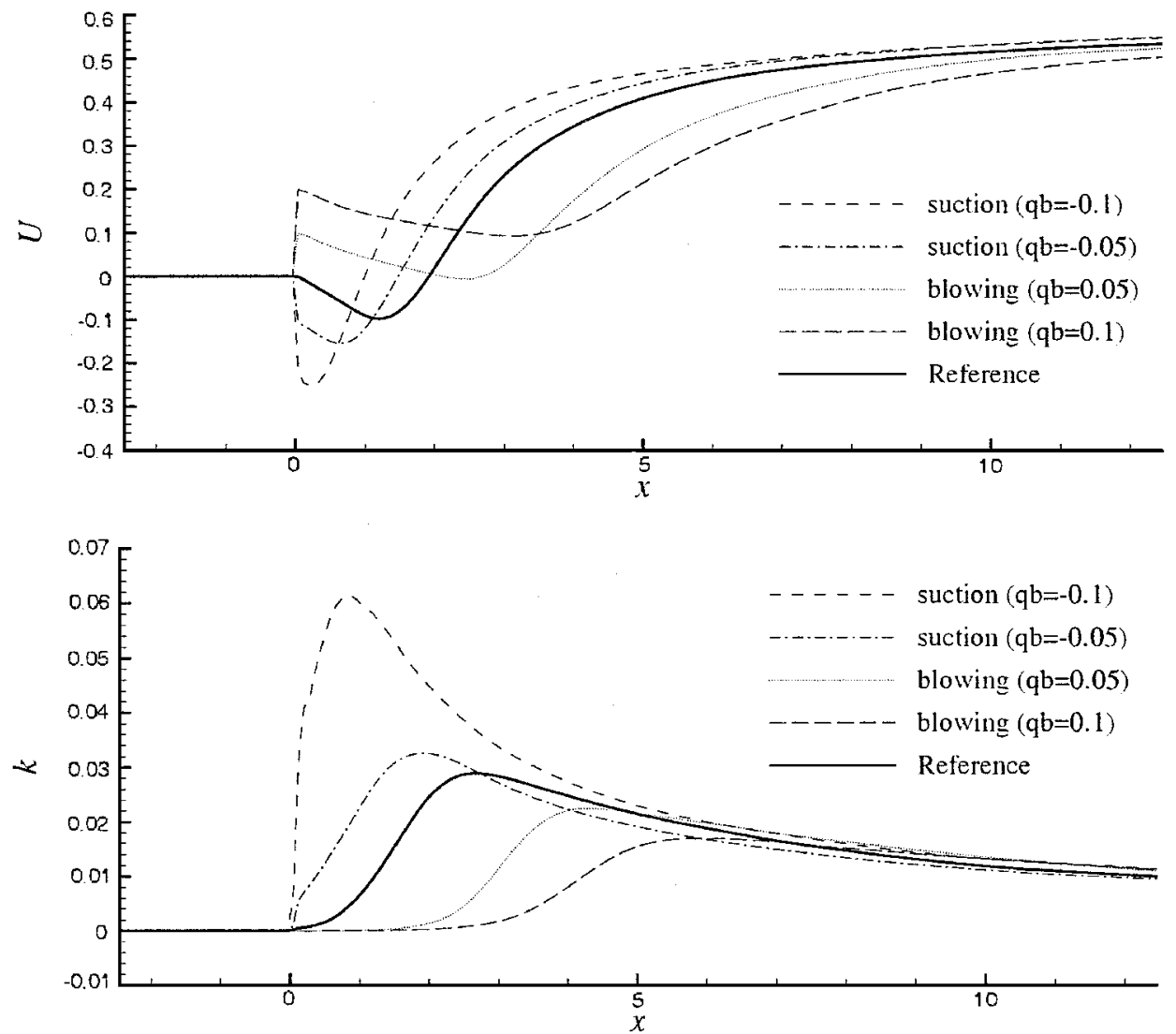

Fig. 8 Mean velocity $U$ and turbulence kinetic energy $k$ distributions for slit height $h_{s}=0.5$; the reference simulation of no flow control is shown with the solid line.

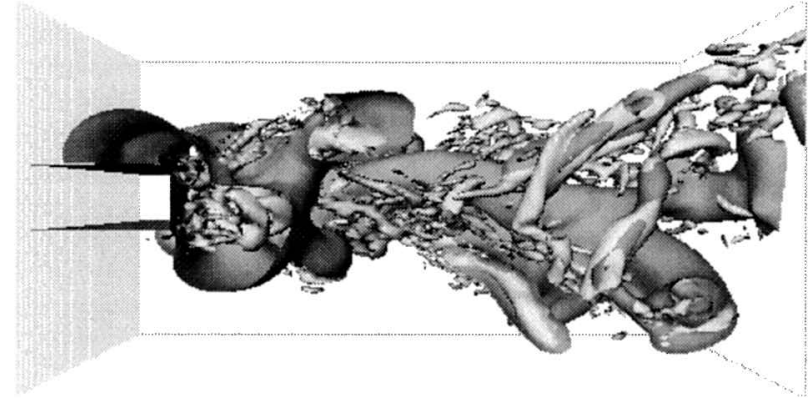

a)

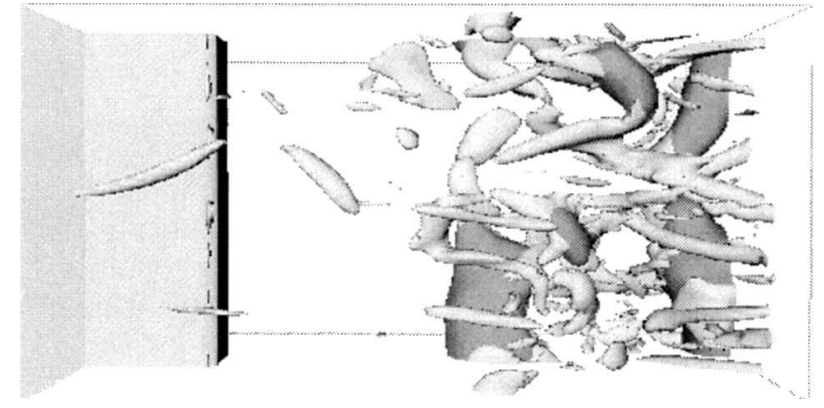

b)

Fig. 9 Instantaneous flow structures in the near-wake region (shade definition as in Fig. 3): a) side view with base suction, $h_{s}=1.0, u_{b}=-0.1$, and $q_{b}=-0.1$ and b) top view with base blowing, $h_{s}=1.0, u_{b}=0.1$, and $q_{b}=0.1$.

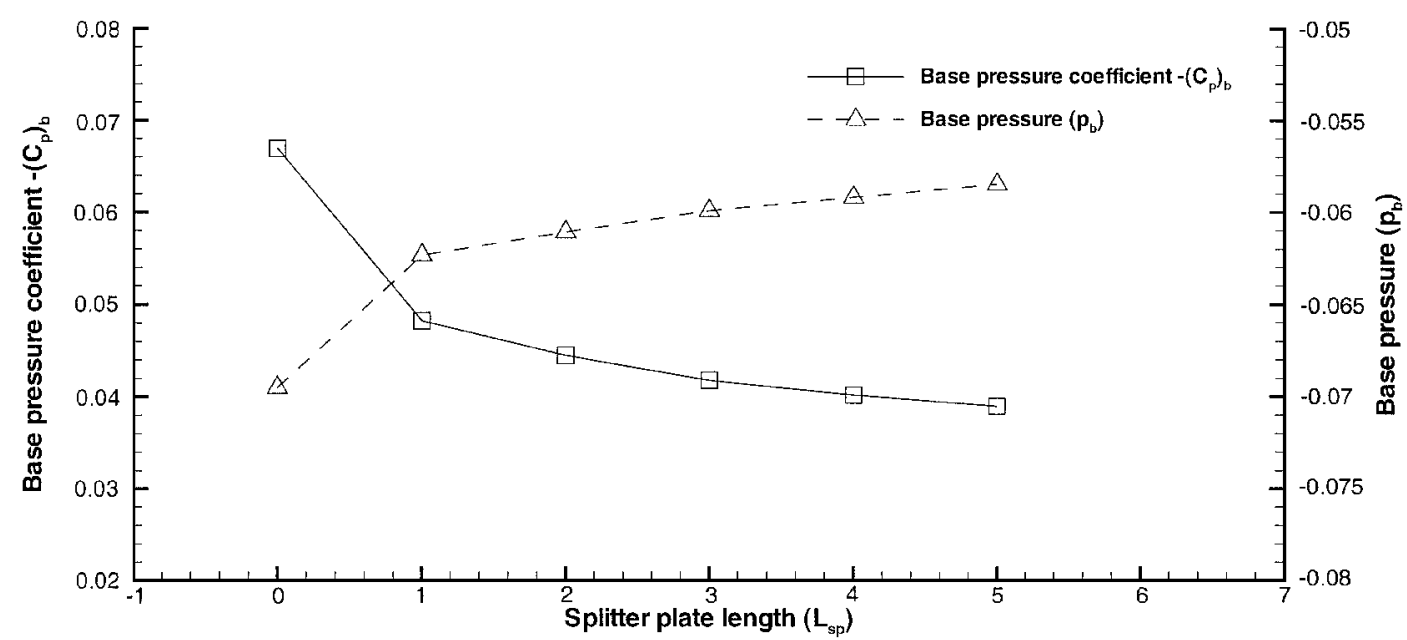

Fig. 10 Base pressure coefficient (left-hand side, square symbols) and base pressure (right-hand side, triangle symbols) as a function of the secondary splitter plate length. 
beyond that was found to be insignificant. A satisfactory physical explanation for the appearance of a local drag minimum has not been offered to date. Experiments and simulations using a wider range of Reynolds number are needed.

Figure 11 shows streamline plots with splitter plate lengths of $L_{\mathrm{sp}}=1$ and 5 . The size of the recirculation grows as the splitter plate length increases, although the bubble shape remains broadly unchanged. A summary of the five cases (cases 11-15) is given in Table 1. Compared to the reference case of no flow control, the separation length $x_{r}$ increases, and the maximum reverse velocity $u_{r}$ decreases, with splitter plate length. The recirculation terminates after the end of the secondary plate for $L_{\mathrm{sp}}=1,2$, and 3 , which agrees with the experimental observation by Bearman, ${ }^{13}$ who claimed that no reattachment was found for shorter splitter plates $L_{\mathrm{sp}}=1 \sim 2$. However, for the longest two plates, the simulated recirculation zone terminates on the plate, that is, the flow behaves broadly as a backward-facing step flow. These two cases have different reattachment lengths, indicating that the flow upstream of reattachment is affected by the flow developments downstream. The recirculation lengths from the simulations $\left(x_{r}=3.91\right.$ for the $L_{\mathrm{sp}}=4$ case and $x_{r}=4.21$ for the $L_{\mathrm{sp}}=5$ case) are longer than experiment, ${ }^{13}$ where a recirculation length of $x_{r}=2.9$ was observed for $L_{\mathrm{sp}}=4$. DNS of a backward-facing step flow ${ }^{21}$ gave a mean value of 6.28 (based on the step height, that is, half of the trailing-edge thickness), equivalent to 3.14 based on the trailing-edge thickness. However, note that the Reynolds number used in the current simulation $\left(5 \times 10^{2}\right.$ based on the equivalent step height) is much lower than that in the experiment, and 10 times lower than that used in the Le et al. ${ }^{21}$ DNS $\left(5.1 \times 10^{3}\right.$ based on the step height). Also, the ratio of the boundary-layer thickness to the step height is close to unity in the study of Le et al., ${ }^{21}$ whereas in the present case the boundary-layer thickness is about 13 times the half-thickness of the trailing edge. Another significant difference to the backward-facing step flow is that no secondary separation was observed in the simulations, which may also be a low Reynolds number effect. The friction drag on the secondary plate was found to be small (6\% at most) compared to the base pressure drag.

Figure 12 gives a comparison of the mean velocity and the turbulence kinetic energy distributions along the centerline. It is clear that even a short splitter plate with a length of $L_{\mathrm{sp}}=1$, that is, equal

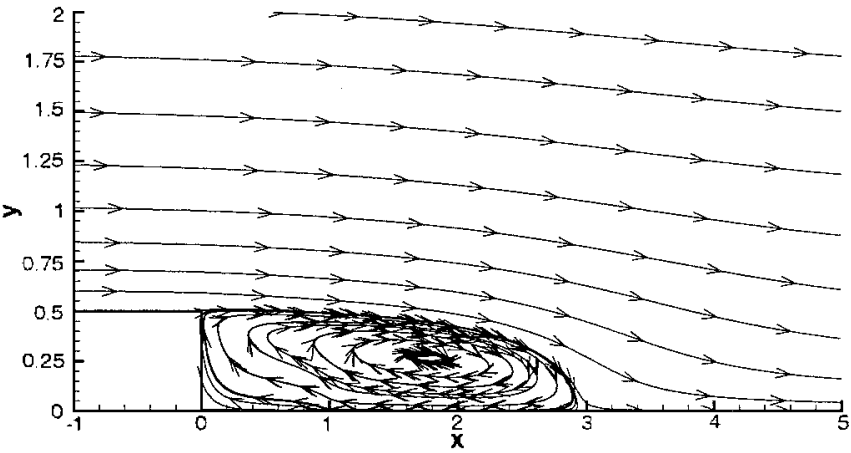

$L_{\mathrm{sp}}=1$

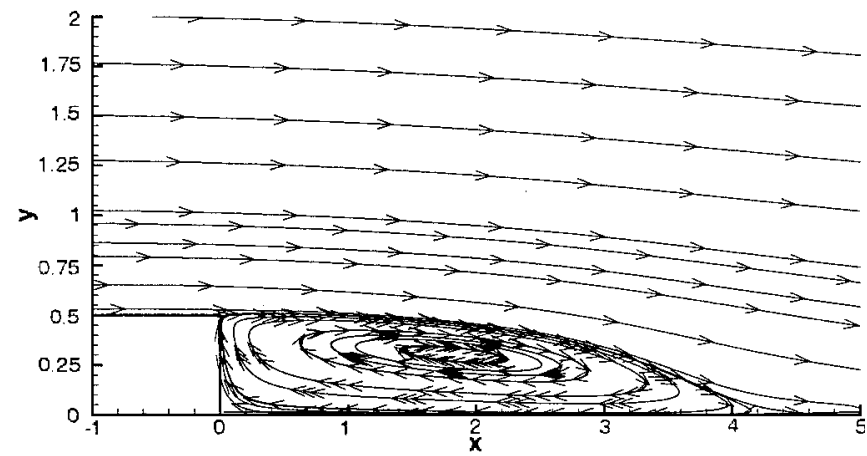

$L_{\text {sp }}=5$

Fig. 11 Streamlines in the near wake with a secondary splitter plate.
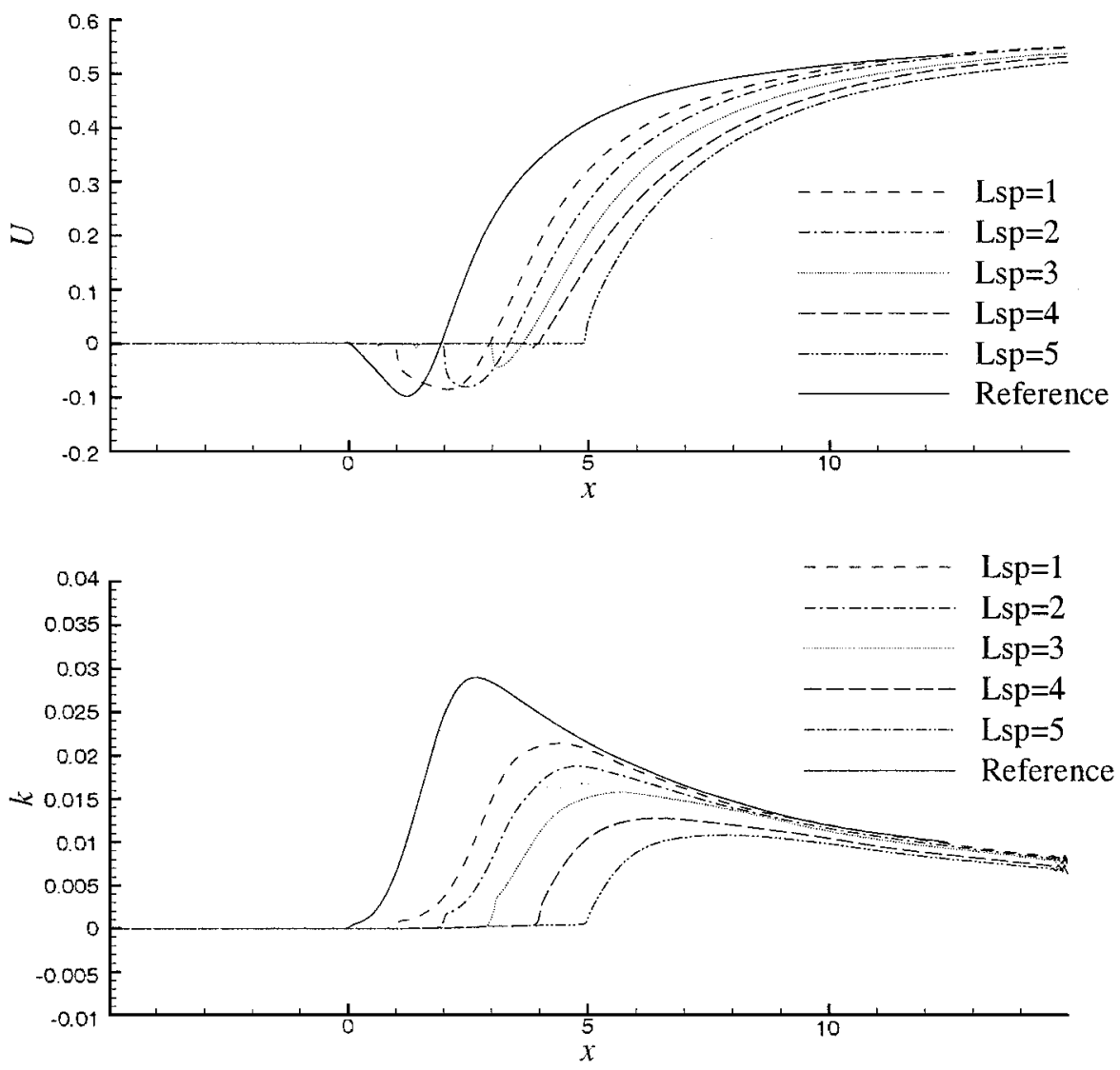

Fig. 12 Mean velocity $U$ and turbulence kinetic energy $k$ distributions of $L_{\mathrm{sp}}=1-5$ compared with the reference simulation. 


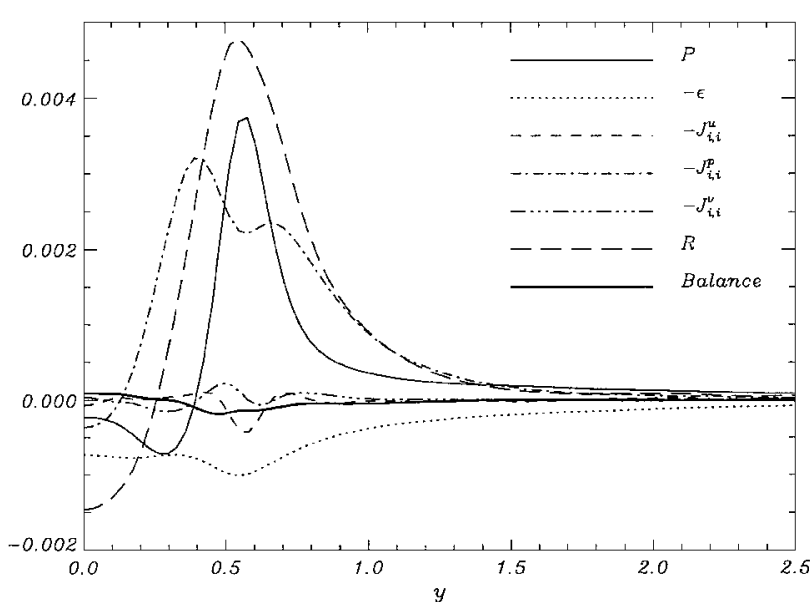

a)

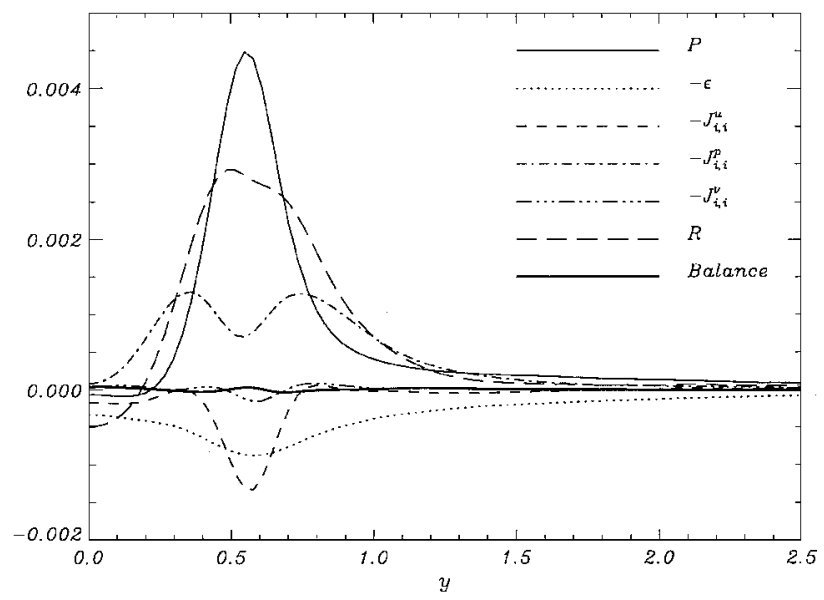

b)

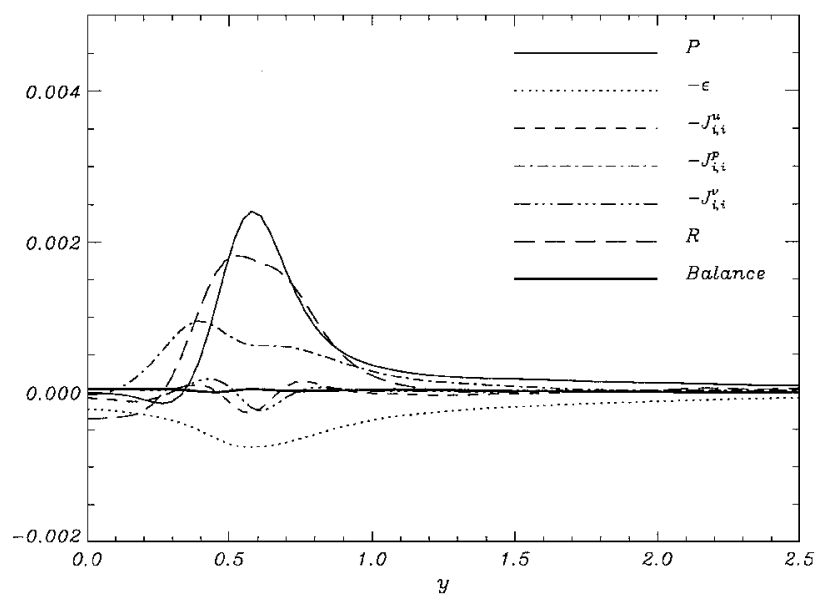

c)

Fig. 13 Turbulence kinetic energy budget in the middle of the recirculation region: a) no flow control, $x=1.0 ; b$ ) base transpiration, $x=1.7$ with $h_{s}=0.25, u_{b}=0.1$, and $q_{b}=0.025$; and c) secondary splitter plate, $x=1.8$ with $L_{\mathrm{sp}}=1$. The thin solid line represents the production term, and the thick solid line represents the balance (sum of all terms).

to the trailing-edge thickness, has a significant effect on the mean velocity and the turbulence kinetic energy distributions.

\section{Energy Budgets}

Energy budgets have been analyzed using statistical data from the simulations. The turbulence kinetic energy equation is

$$
\frac{\partial k}{\partial t}+R=P-\epsilon-\frac{\partial\left(J_{i}^{u}+J_{i}^{p}+J_{i}^{v}\right)}{\partial x_{i}}
$$

where $R=\left\langle u_{i}\right\rangle\left(\partial k / \partial x_{i}\right)$ is the convection term; $P$ is the production term; $\epsilon$ is the dissipation; and $J_{i}^{u}, J_{i}^{p}$, and $J_{i}^{v}$ are turbulence

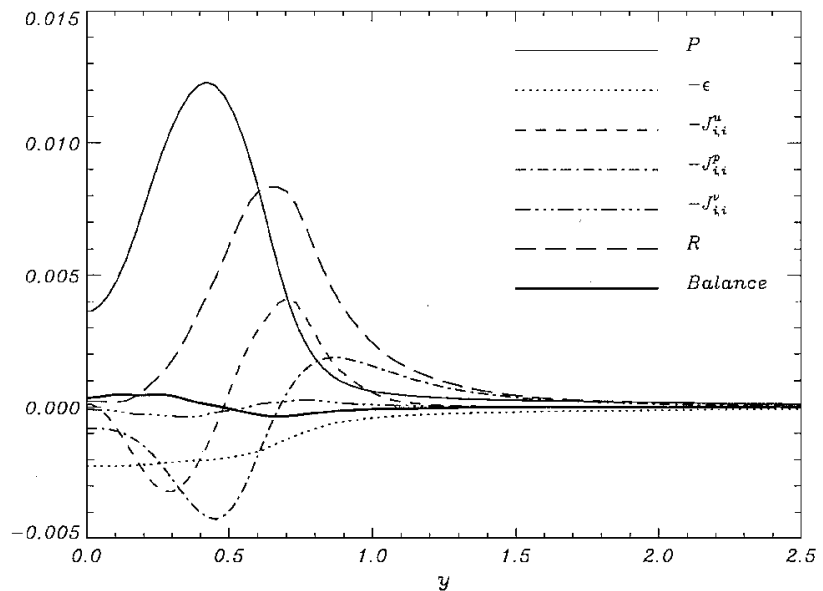

a)

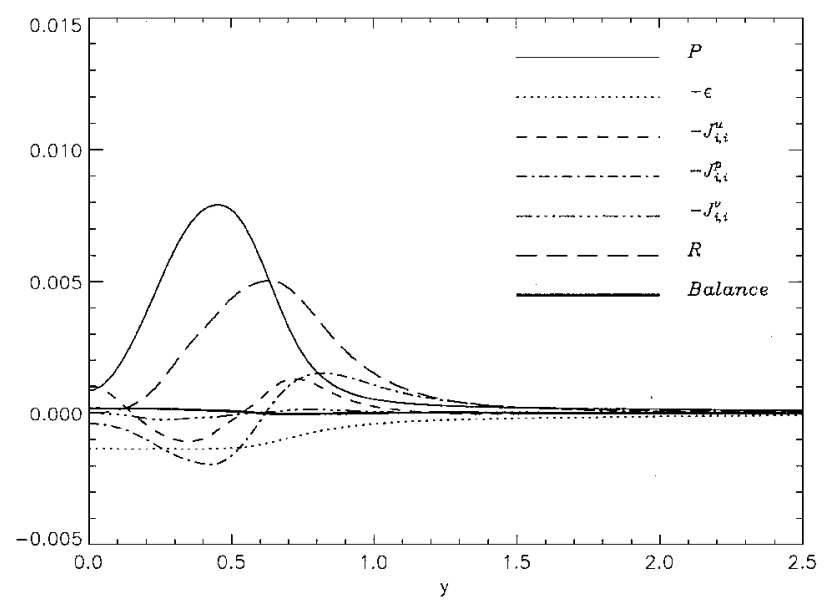

b)

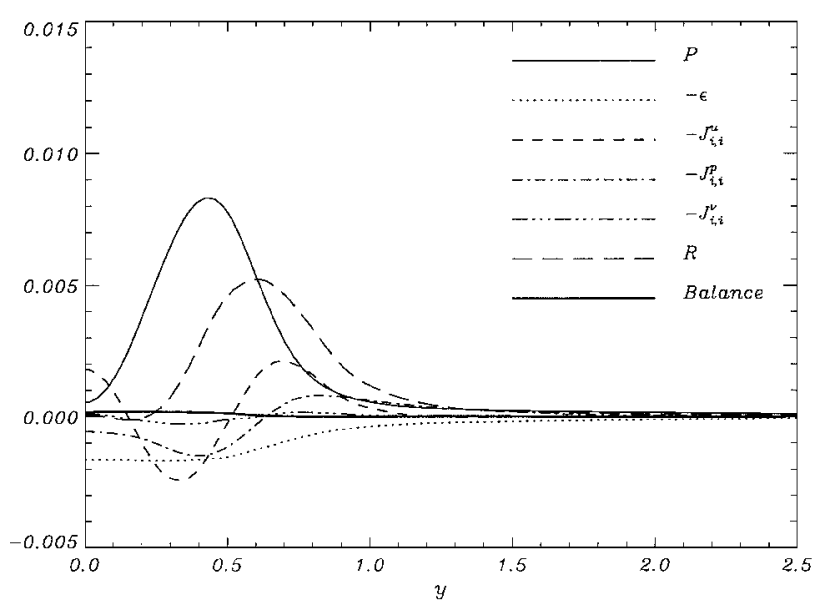

c)

Fig. 14 Turbulence kinetic energy budget at the end of the recirculation region: a) no flow control, $x=2.0 ;$ b) base transpiration, $x=2.5$ with $h_{s}=$ $0.25, u_{b}=0.1$, and $q_{b}=0.025$; and c) secondary splitter plate, $x=2.9$ with $L_{\mathrm{sp}}=1$. The thin solid line represents the production term, and the thick solid line represents the balance (sum of all terms).

triple-moment(transport), pressure-diffusion, and viscous transport terms, respectively, defined in detail by Yao et al. ${ }^{1}$

Turbulence kinetic energy budgets were computed for three different cases: no flow control, flow control with base transpiration $\left(h_{s}=0.25, u_{b}=0.1\right.$, and $\left.q_{b}=0.025\right)$, and flow control with secondary splitter plate $\left(L_{\mathrm{sp}}=1\right)$. The comparisons were carried out at two selected positions: the middle of the recirculation region and the end of the recirculation region. Results are shown in Figs. 13 and 14.

At the middle of the recirculation region, for the reference case of no flow control (Fig. 13a), the convection term is very significant 
with a positive peak at $y \simeq 0.5$ and a negative peak (correspondingto a reverse flow) at the centerline, $y=0$. The production term also has a small negative peak near the centerline and a high positive peak away from it. The negative production was studied by Thomas et al. ${ }^{20}$ A strong pressure-diffusionterm, with the same order of magnitude as the production and convection terms, is also observed. It implies that the pressure-diffusioneffect cannot be neglected in the trailingedge flow. The dissipation has a maximum at the same position as the maximum production but is only approximately $30 \%$ of the peak production value. Hence, the flow is locally far from an equilibrium state. When base transpiration is applied (Fig. 13b), the production and dissipation terms remain broadly unchanged, whereas the convection and pressure-diffusion terms are both reduced. However, a significant negative peak in turbulence triple-moment (transport) term appears, again at the same peak position of the production and dissipation terms. With the secondary splitter plate (Fig. 13c), a significant decrease in the peak production (almost half of the value compared to that in the reference case) is evident, whereas the other terms are, with the exception of the triple-moment term, similar to those found in the base transpiration case.

At the end of the recirculation region, for the case of no flow control (Fig. 14a), the production term dominates the energy budget, with significant contributions from the convection, turbulence triple-moment, pressure-diffusion, and dissipation terms. The pressure diffusion changes from positive to negative as the centerline is approached.For the two types of flow control considered (Figs. 14b and $14 \mathrm{c}$ ), the peak value of all terms decreases, with the pressure diffusion being more significantly affected than the other terms. The triple-moment term is relatively less important for the base transpiration (blowing) case than for either the reference case or for the secondary splitter plate case, $L_{\mathrm{sp}}=1$.

The detailed behavior of terms in the energy budget discussedearlier is important for constructing models at the two-equation level, which can subsequently be used for higher Reynolds number studies and optimization of control schemes. However, the main results can be summarized more simply. At the end of the recirculation zone (Figs. 14b and 14c) we are seeing the result of flow control, in that all turbulence activity is diminished, whereas in the middle of the recirculation we see differences in detail between the two control schemes. In particular, the secondary splitter plate method is more effective at reducing turbulence activity within the recirculation zone.

\section{Conclusions}

It can be concluded from this study that DNS is feasible for the parametric study of flow control in the trailing-edgeflow, using base transpiration and a secondary splitter plate. Both methods of flow control have a significant effect on the aerodynamic performance, measured, for example, by the base pressure coefficient. Simulations with base transpiration have been carried out with different slit heights and volume flow rates. It was revealed from the simulation that suction (even weak suction) enhances the near-wake vortex shedding, sustains the coherent structure over an extended region, and shortens the recirculationregion, whereas strong blowing moves the entire interaction downstream and diminishes the coherentstructure more quickly. It was also found that for the same volume flow rate, a greater base pressure increase can be obtained by blowing slowly through a wide slit rather than quickly through a narrow slit, in agreement with experiments. Simulations with secondary splitter plate showed a monotonic increase in base pressure coefficient with the splitter plate length. A $25 \%$ increase was achieved for the splitter plate equal to the trailing-edge thickness, whereas a maximum $44 \%$ increase was achieved for the splitter plate length five times the trailing-edge thickness. Note that in practical applications, the base pressure drag is not the only consideration, and the drag reduction problem must be considered in the context of aerodynamic and structural constraints. The turbulence kinetic energy budget in the recirculation region shows that the pressure-diffusionterm has a significant contribution to the budget balance, whereas the peak of dissipation is only about $30 \%$ of the peak production. The effect of flow control is, in general, to reduce the convection and pressure transport relative to the production in the core of the recirculation. At the end of the recirculation region, the structure of the budget is retained, but the magnitudes of all terms are reduced.

\section{Acknowledgments}

The authors would like to thank the United Kingdom Engineering and Physical Science Research Council for financial and parallel computer support through Grants GR/L 18570 and GR/M 08424.

\section{References}

${ }^{1}$ Yao, Y. F., Thomas, T. G., Sandham, N. D., and Williams, J. J. R., "Direct Numerical Simulation of Turbulent Flow over a Rectangular Trailing Edge," Theoretical and ComputationalFluid Dynamics, Vol. 14, No. 5, 2001, pp. 323-336.

${ }^{2}$ Nash, J. F., "A Discussion of Two-Dimensional Turbulent Base Flows," Aeronautical Research Council, TR R\&M 3468, London, 1967.

${ }^{3}$ Wood, C. J., "The Effect of Base Bleed on a Periodic Wake," Journal of the Royal Aeronautical Society, Vol. 68, July 1964, pp. 477-482.

${ }^{4}$ Bearman, P. W., "The Effect of Base Bleed on the Flow Behind a TwoDimensional Model with a Blunt Trailing Edge," Aeronautical Quarterly, Vol. 18, Aug. 1967, pp. 207-224.

${ }^{5}$ Motallebi, F., and Norbury, J. F., "Effect of Base Bleed on Vortex Shedding and Base Pressure in Compressible Flow," Journal of Fluid Mechanics, Vol. 110, 1981, pp. 273-292.

${ }^{6}$ Deckers, M., and Denton, J. D., "Aerodynamics of Trailing-Edge-Cooled Transonic Turbine Blades: Part 1-Experimental Approach,” American Society of Mechanical Engineers, ASME Paper 97-GT-518, 1997

${ }^{7}$ Pappu, K. R., and Schobeiri, M. T., "Optimization of Trailing Edge Ejection Mixing Losses: A Theoretical and Experimental Study," American Society of Mechanical Engineers, ASME Paper 97-GT-523, 1997.

${ }^{8}$ Bearman, P. W., "Near Wake Flows Behind Two- and Three-Dimensional Bluff Bodies," Journal of Wind Engineering and Industrial Aerodynamics, Vol. 49, No. 71, 1997, pp. 33-54.

${ }^{9}$ Hannemann, K., and Oertel, H., "Numerical Simulation of the Absolutely and Convectively Unstable Wake," Journal of Fluid Mechanics, Vol. 199, 1989, pp. 55-88.

${ }^{10}$ Hammond, D. A., and Redekopp, L. G., "Global Dynamics and Aerodynamic Flow Vectoring of Wakes," Journal of Fluid Mechanics, Vol. 338 , 1997, pp. 231-248.

${ }^{11}$ Roshko, A., "On the Wake and Drag of Blunt Bodies," Journal of Aerospace Sciences, Vol. 22, Feb. 1955, pp. 124-132.

${ }^{12}$ Nash, J. F., Quincey, V. G., and Callinan, J., "Experiments on TwoDimensional Base Flow at Subsonic and Transonic Speeds," Aeronautica Research Council, TR R\&M 3427, London, 1963.

${ }^{13}$ Bearman, P. W., "Investigation of the Flow Behind a Two-Dimensional Model with a Blunt Trailing Edge and Fitted with Splitter Plates," Journal of Fluid Mechanics, Vol. 21, 1965, pp. 241-255.

${ }^{14}$ Tanner, M., "Reduction of Base Drag," Progress in Aerospace Sciences, Vol. 16, No. 4, 1975, pp. 369-384.

${ }^{15}$ Rathakrishnan, E., "Effect of Splitter Plate on Bluff Body Drag," AIAA Journal, Vol. 37, No. 9, 1999, pp. 1125, 1126

${ }^{16}$ Park, W. C., and Higuchi, H., "Numerical Investigation of Wake Flow Control by a Splitter Plate," KSME International Journal, Vol. 12, No. 1, 1998, pp. 123-131.

${ }^{17}$ Lund, T. S., Wu, X., and Squires, K. D., "Generation of Turbulent Inflow Conditions for Boundary Layer Simulations," Journal of Computational Physics, Vol. 140, No. 2, 1998, pp. 233-258.

${ }^{18}$ Thomas, T. G., and Williams, J. J. R., "Development of a Parallel Code to Simulation Skewed Flow over a Bluff Body," Journal of Wind Engineering and Industrial Engineering, Vol. 67-68, April-June 1997, pp. 155-167.

${ }^{19}$ Gough, T. D., and Hancock, P. E., "Lower Reynolds Number Turbulent Near Wakes," Advances in Turbulence VI, edited by S. Gavrilakis, L. Machiels, and P. A. Monkewitz, Kluwer Academic, Norwell, MA, 1996 , pp. $445-448$.

${ }^{20}$ Thomas, T. G., Yao, Y. F., and Sandham, N. D., "Structure and Energetics of a Turbulent Trailing Edge Flow," Computers and Mathematics with Applications (to be published).

${ }^{21}$ Le, H., Moin, P., and Kim, J., "Direct Numerical Simulation of Turbulent Flow over a Backward-Facing Step," Journal of Fluid Mechanics, Vol. 330, 1997, pp. 349-374.

W. J. Devenport Associate Editor 